\title{
The Critical Properties of Anisontropic Ising Model with Competing Interactions in the Region of Transition from Modulated Phase into Paramagnetic
}

\author{
Murtazaev Akai Kurbanovich ${ }^{1,2, *}$, Ibaev Zhavrail Gadzhievich ${ }^{1}$ \\ ${ }^{1}$ Institute of Physics, Daghestan Scientific Center, Russian Academy of Sciences, Russia \\ ${ }^{2}$ Department of Physics, Dagestan State University, Russia
}

Copyright $(2016$ by authors, all rights reserved. Authors agree that this article remains permanently open access under the terms of the Creative Commons Attribution License 4.0 International License

\begin{abstract}
The anisotropic Ising model with competing interactions in the region of transition from a modulated phase into paramagnetic state is investigated by the Monte-Carlo methods. By means of histogram analysis and the finite-size scaling method, the modulated - paramagnetic phase transition is shown to be a second order phase transition. Critical parameters and temperatures of phase transitions in this region are calculated.
\end{abstract}

Keywords A Modulated Ordering, ANNNI-model, Lifshitz Point, Monte-Carlo Methods, Phase Transition, Critical Properties

\section{Introduction}

In addition to simple magnetic orderings (ferromagnetic, antiferromagnetic, or ferrimagnetic), in nature there is a new type of magnetic ordering - modulated. Such ordering appears in systems with an exchange competition independently from a physical nature of this interaction. A number of real materials, in which the modulated ordering is possible, have long risen beyond 100 [1-3].

We note that depending on the correlation of parameters of competing interactions and a temperature, such systems may show different kinds of magnetic ordering (ferromagnetic, modulated, paramagnetic). Correspondingly, there are possible several different phase transitions in the systems. For instance, the transitions from a homogenous state into the modulated one, from the modulated phase into a paramagnetic state, and transitions in the modulated phase between different modulated structures may occur in modulated magnetic ordering systems. Experimental and theoretical study of phase transitions and critical phenomena is rather a difficult problem even for such simple cases as transitions from ferromagnetic state into the paramagnetic phase. Therefore, today the most acceptable and precise description of phase transitions and critical phenomena is provided only by computer calculations using the Monte-Carlo and molecular dynamics methods.

In practice, the systems with possible modulated magnetic ordering are studied using different models. The simplest and valid among them is an anisotropic Ising model with competing interactions (ANNNI - model, Fig. 1).

\section{Model}

ANNNI - model appeared in the statistic physics in the latter half of $20^{\text {th }}$ century to interpret a spiral magnetic ordering in rear-earth metals [4-7]. The Hamiltonian of the model on a regular cubic lattice is:

$$
H_{A N N N I}=-J \sum_{i, j} s_{i} s_{j}+J_{1} \sum_{i} s_{i} s_{i+1},
$$

where $s_{i}= \pm 1, J$ is the parameter of exchange interaction of neighbor spin pairs, $J_{1}>0$ is the parameter of antiferromagnetic interaction of next nearest neighbors along $\mathrm{Z}$ axis.

ANNNI - model is precisely solved only for one-dimensional case [8]. Nevertheless, some of its peculiarities are defined for large dimensions well enough. For three-dimensional (3D) case, a topology $T-\left|J_{l} / J\right|$ of a phase diagram includes three areas intersecting in a multicritical Lifshitz point [9, 10]. The system is paramagnetic at high temperatures and ferromagnetic at lower $T$ and small $\left|J_{l} / J\right|$. When $\left|J_{l} / J\right|$ has sufficiently large values, the modulated phases take place (Fig. 2). 


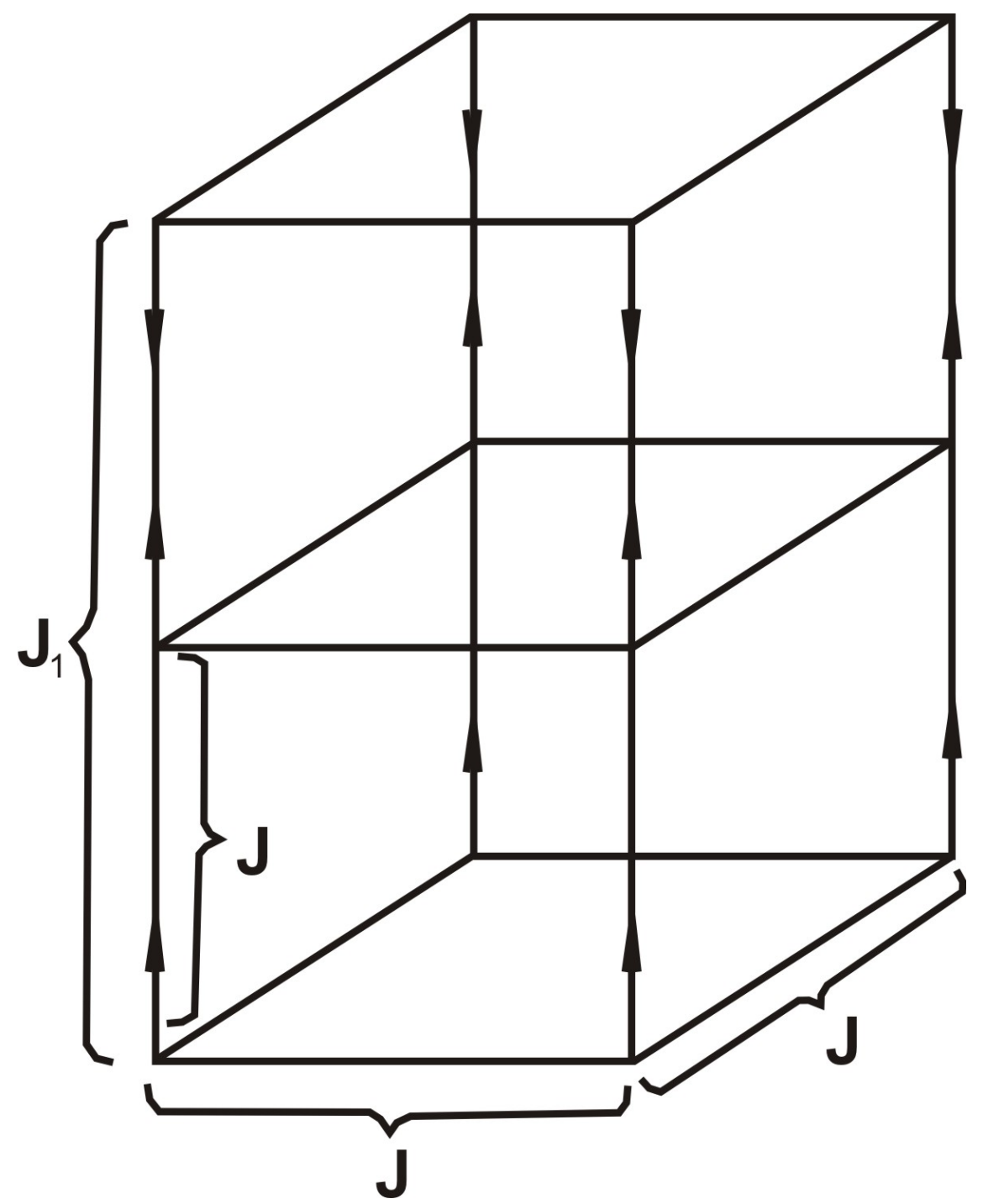

Figure 1. Anisotropic model with competing interactions

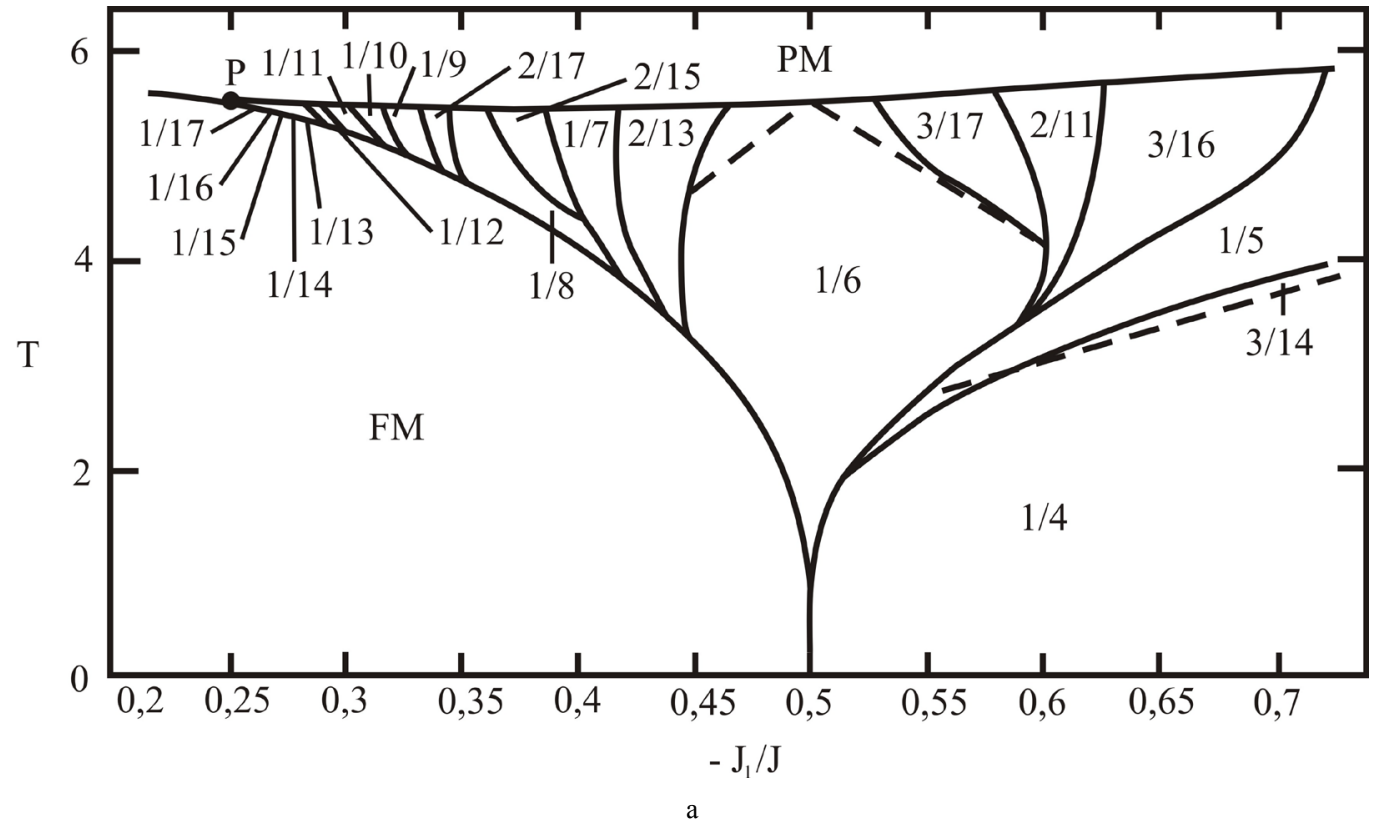




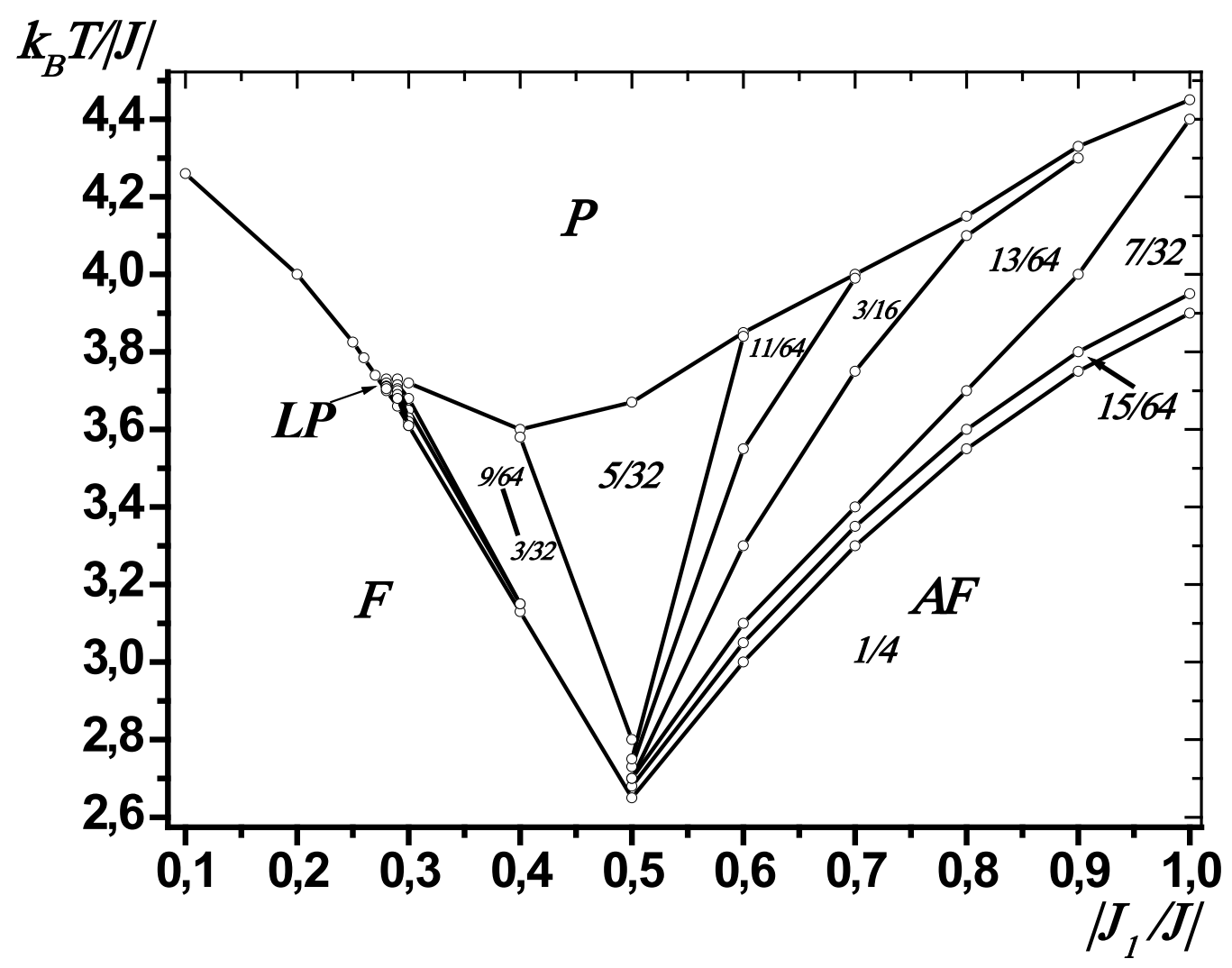

b

Figure 2. The phase diagram of anisotropic Ising model with competing interactions: a) by data from [15]; b) by data from [10].

The phase behavior of studied model has been described using approximated theoretical methods, including high- and low-temperature expansions $[12,13]$, the mean field theory [14] and other theoretical approximations [15-17].

In accordance to literature data, the ANNNI - model undergoes a second order transition from paramagnetic state into the nearest ordered state, and ferromagnetic - modulated phase transition is a first order transition [11].

A Monte-Carlo simulation [8, 18-22] is made for values of $\left|J_{l} / J\right|=0.1 \div 1.0$ including the multicritical region of Lifshitz point [23]. Critical parameters of the second order transition from ferromagnetic phase into paramagnetic phase are calculated, the multicritical behavior of the system in the vicinity of Lifshitz point is described.

The recent more precise critical exponents in the vicinity of the Lifshitz point are obtained by means of the anisotropic scaling [22] and Monte - Carlo methods [23].

According to data [21-26], the critical behavior of modulated structure systems at low values of $\left|J_{I} / J\right|$ is described by the Ising universality class of a critical behavior. With increase in $\left|J_{I} / J\right|$, a crossover to the critical behavior of multicritical Lifshitz point and from it to XY class of the critical behavior [24-26] occurs.

In recent work [27], ANNNI - models were studied by the Monte-Carlo methods using an averaged amplitude value of the modulated structure as the order parameter. Obtained results showed that a transition from modulated phase into paramagnetic state is the second order phase transition. In present work, we report the data of the histogram analysis and the values of critical parameters for the anisotropic Ising model with competing interactions at the transition from the modulated state into paramagnetic state.

\section{Investigation Method and Results}

For investigation of critical phenomena we used the cubic systems with periodic boundary conditions and sizes $L \times L \times L ; L=12 \div 64$. A number of spins in simulated systems was $N_{\text {eff }}=512 \div 262144$. The Markovian chains of $\tau=1000 \tau_{0} \quad\left(\tau_{0}=10^{4} \mathrm{MC}\right.$ step/spin is the length of a nonequilibrium part) were generated in a computer. Thermodynamic parameters of the system were calculated by the averaging along the chain. In addition, the averaging over thousand different initial configurations were done. The error of obtained results did not exceed the sizes of signs used in figures.

Note that modern computational physics fails to design an algorithm for more precise investigation of ANNNI model in the whole area of change in $\left|J_{I} / J\right|$ and $T$ parameters. Although the high-precision cluster algorithms, in particular the one-cluster Wolf algorithm [28], allows studying of the system in the region of the second order transition, virtually it doesn't provide any results in the region of modulated phases and the nearest vicinity of Lifshitz point. The modifications of known algorithms offered in the literature $[27,29]$ serve to solve minor subproblems of the model. The classical Metropolis algorithm is a sole algorithm of 
Monte-Carlo method which doesn't become inoperative for this model in any interval of change in $\left|J_{I} / J\right|$ and $T$ parameters. Though an algorithm precision is not high, the obtained results help to realize the pattern of thermodynamic and critical behavior of ANNNI - model.

We received temperature dependences of the heat capacity and susceptibility using the expressions [14]:

$$
\begin{gathered}
C=\left(N K^{2}\right)\left(\left\langle U^{2}\right\rangle-\langle U\rangle^{2}\right) \\
\chi=(N K)\left(\left\langle m^{2}\right\rangle-\langle m\rangle^{2}\right),
\end{gathered}
$$

where $K=|J| / k_{B} T ; U$ is the intrinsic energy; $m$ is the order parameter.

For investigation of magnetic properties of modulated region, we used a value

$$
M_{Z}=\frac{1}{L^{2}} \sum_{x, y}^{L} S_{x, y, z},
$$

which is the average magnetization of a layer perpendicular to $\mathrm{Z}$ axes.

The wave number values and a character of amplitude and phase modulation were defined using a mathematical set of spectrum analysis based on Fourier transforms [30]

$$
\begin{aligned}
& M(z)=\frac{a_{0}}{2}+ \\
& +\sum_{k=1}^{N}\left[a_{k} \cos (2 \pi k z / L)+b_{k} \sin (2 \pi k z / L)\right.
\end{aligned}
$$

where

$$
a_{0}=\frac{1}{L} \sum_{z=1}^{L} M_{Z}
$$

$$
\begin{gathered}
a_{k}=\frac{2}{L} \sum_{z=1}^{L} M_{Z} \cos (2 \pi k z / L), \\
b_{k}=\frac{2}{L} \sum_{z=1}^{L} M_{Z} \sin (2 \pi k z / L), \\
q=k / L
\end{gathered}
$$

$k$ is the number of harmonics.

As the modulated structures are harmonic the wave number $q$ is detected by a $k$ value at which the amplitude function $\left(a_{k}^{2}+b_{k}^{2}\right)$ has a maximum. Figure 3 exhibits a characteristic modulated structure, appearing in the system with $\left|J_{l} / J\right|=0.7$ at $\mathrm{T}=3.4$, and its Fourier transform diagram.

For study the properties of the modulated - paramagnetic phase transition, we used the histogram analysis and the finite-size scaling theory. The application of these methods allows determination of a phase transition kind.

In the histogram analysis of data [31], a possibility to find a system with the $U$ value and an order parameter $m$ is determined by an expression:

$$
p(U, m)=\frac{1}{Z(K)} W(U, m) \exp (K U)
$$

where $W(U, m)$ is a number of configurations with the $U$ and order parameter $m, Z(K)$ is the energy distribution of the whole system. The results of the histogram analysis of ANNNI - model at modulated - paramagnetic transition for $\left|J_{l} / J\right|=0.7$ are shown in Figure 4. As is evident from Figure 4, one well-marked maximum is observed on the energy distribution probability for two values on linear sizes $L=32$ and 64. Such a behavior of $p(U, m)$ indicates that the modulated - paramagnetic state transition is the second order phase transition. 

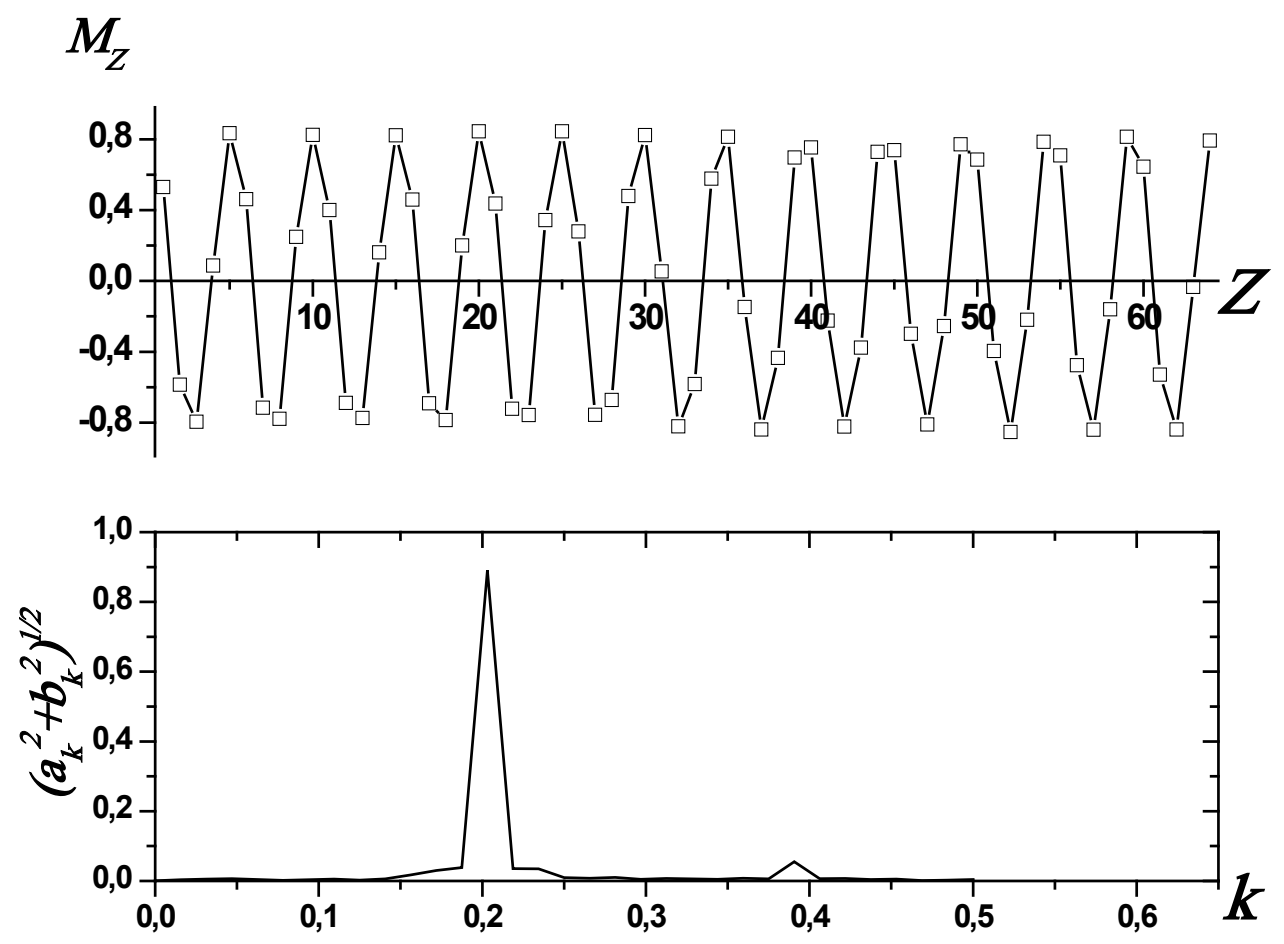

Figure 3. The characteristic modulated magnetic structure and the amplitude of its Fourier transform $\left(\mathrm{T}=3.4\right.$ and $\left.\left|J_{I} / J\right|=0.7\right)$.

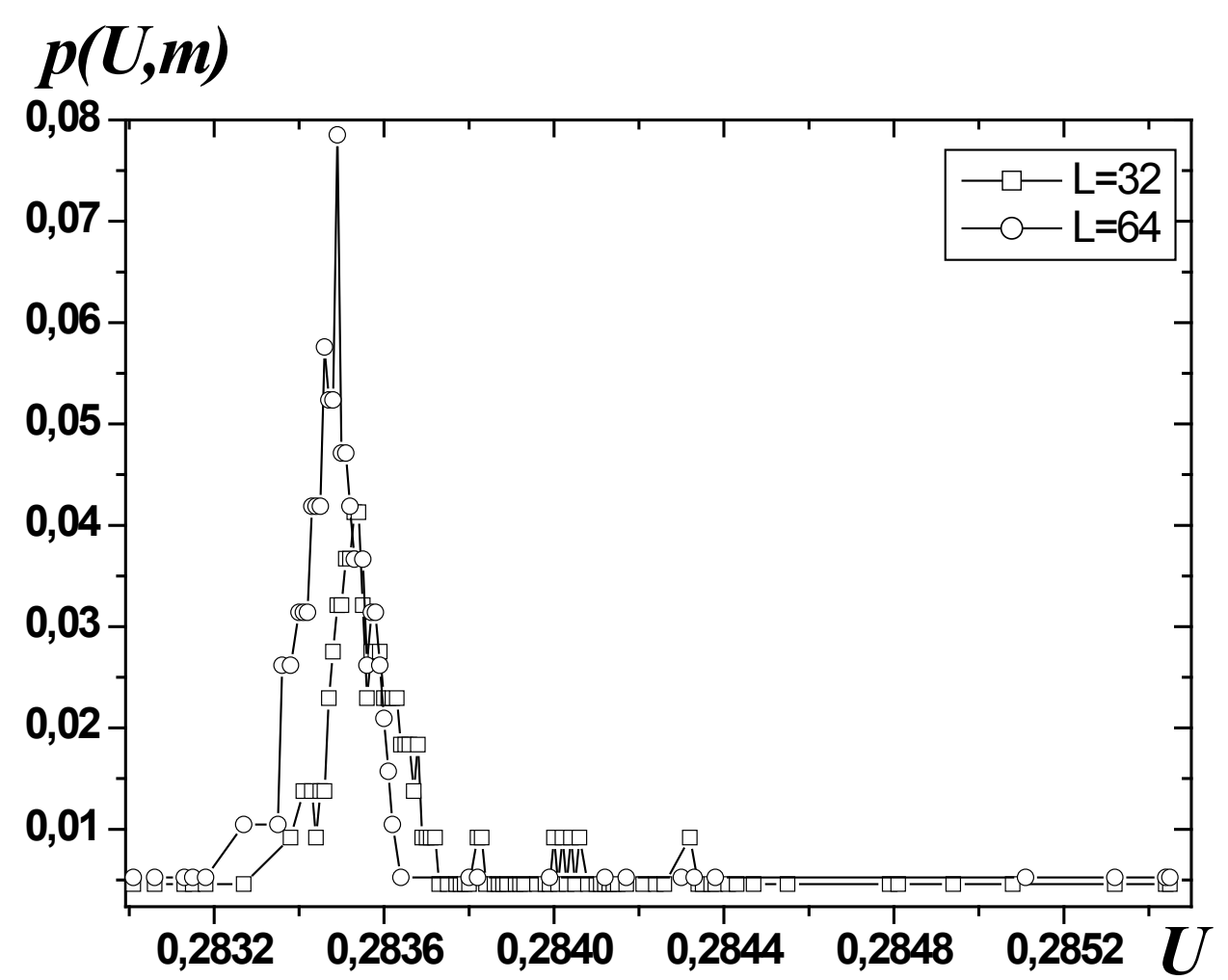

Figure 4. The histogram of the energy distribution of ANNNI - model for $\left|J_{1} / J\right|=0.7$ at phase transition from modulated state into paramagnetic state.

The critical temperature was estimated using the methods of Binder cumulants. According to [32], the cumulants

$$
\left.U_{L}=1-\left\langle m^{4}\right\rangle / 3 m^{2}\right\rangle^{2}
$$

for the systems different sizes $L$ are intersects in a critical point $T_{c}$. Plotting the $U_{L}$-temperature dependences for the systems with different sizes $L$ we can calculate the critical temperatures of systems. The values of critical temperatures for different values of $\left|J_{I} / J\right|$ calculated by this method are presented in Table 1.

The correlations of the finite-size scaling theory were used 
for estimation of the critical parameters of the order parameter, susceptibility, and correlation radius [33-36]. From these correlations, it follows that

$$
\begin{gathered}
m \sim L^{-\beta / v}, \\
\chi \sim L^{\gamma / v}, \\
V_{n}=L^{1 / v} g_{V_{n}},
\end{gathered}
$$

at $T=T_{c}$ and sufficiently large $L$, where $g_{V_{n}}$ is a some constant, as $V_{n}$ can serve

$$
V_{i}=\frac{\left\langle m^{i} E\right\rangle}{\left\langle m^{i}\right\rangle}-\langle E\rangle, i=1,2,3 \ldots
$$

The heat capacity was approximated by an expression

$$
C_{\max }(L)=C_{\max }(L=\infty)-a L^{\alpha / v},
$$

where $a$ is a some coefficient.

To determine the critical exponents we plotted the log-log diagrams of system linear size - thermodynamic parameter dependences at $T=T_{c}$. Figures 5-8 present the characteristic dependences of order parameter, susceptibility, heat capacity, and $V_{i}$ parameters at $i=1,2,3$ on the linear sizes of the lattice at $\left|J_{I} / J\right|=0.7$. As is obvious from the Figures, all points in diagrams are good fall on a line within an error. The inclination of line in Figures 4-7 defines the values of $\beta / v, \gamma / v$, $\alpha / v$ and $1 / v$. The obtained values of critical exponents are presented in Table 1.

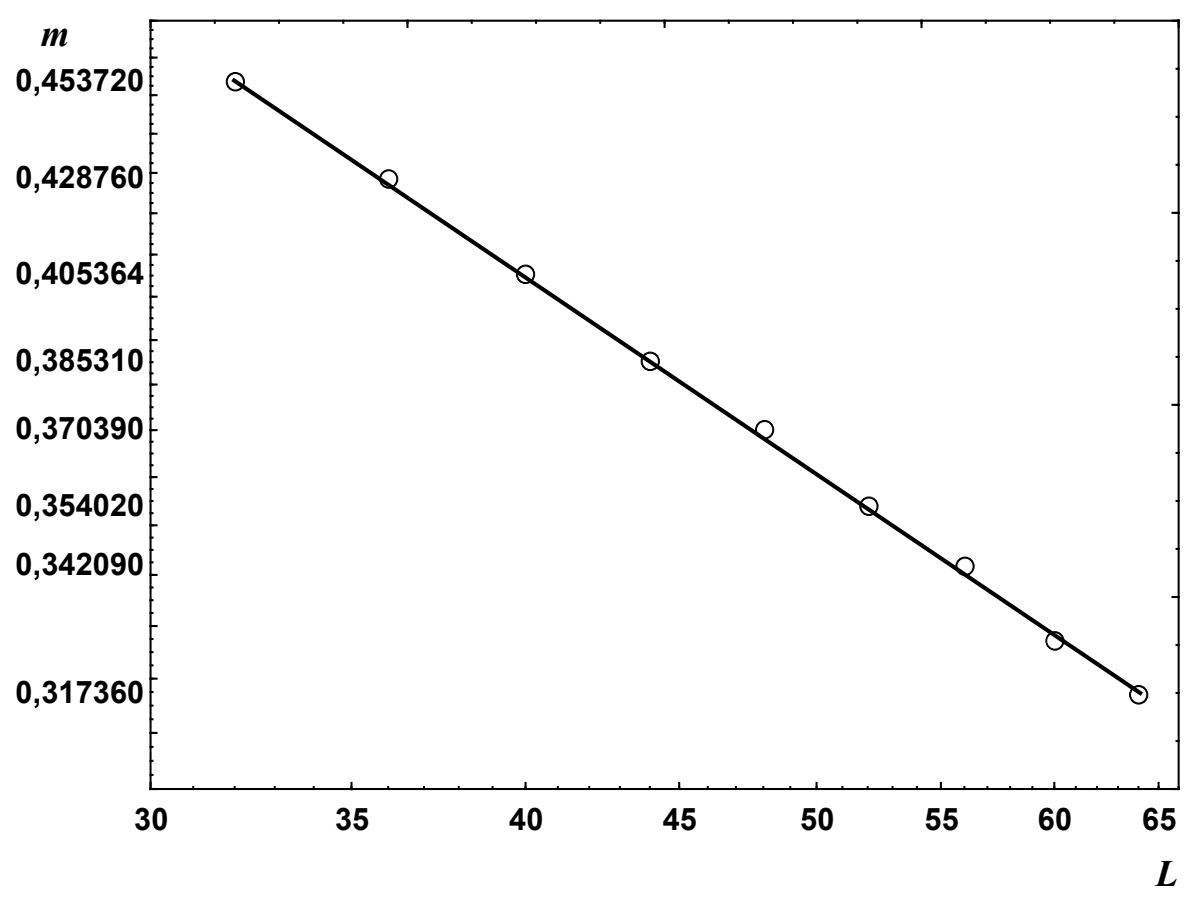

Figure 5. The log-log dependence of the magnetization. 


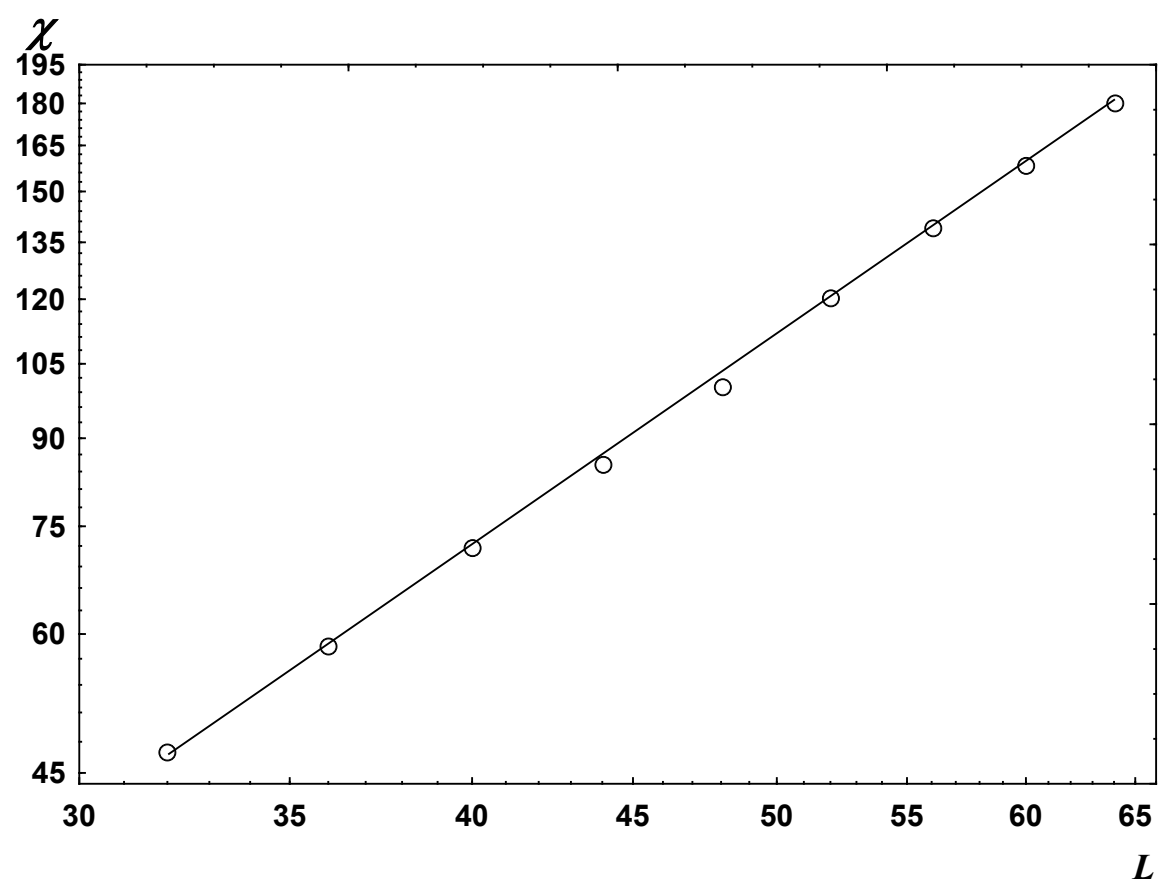

Figure 6. The log-log dependence of the susceptibility.

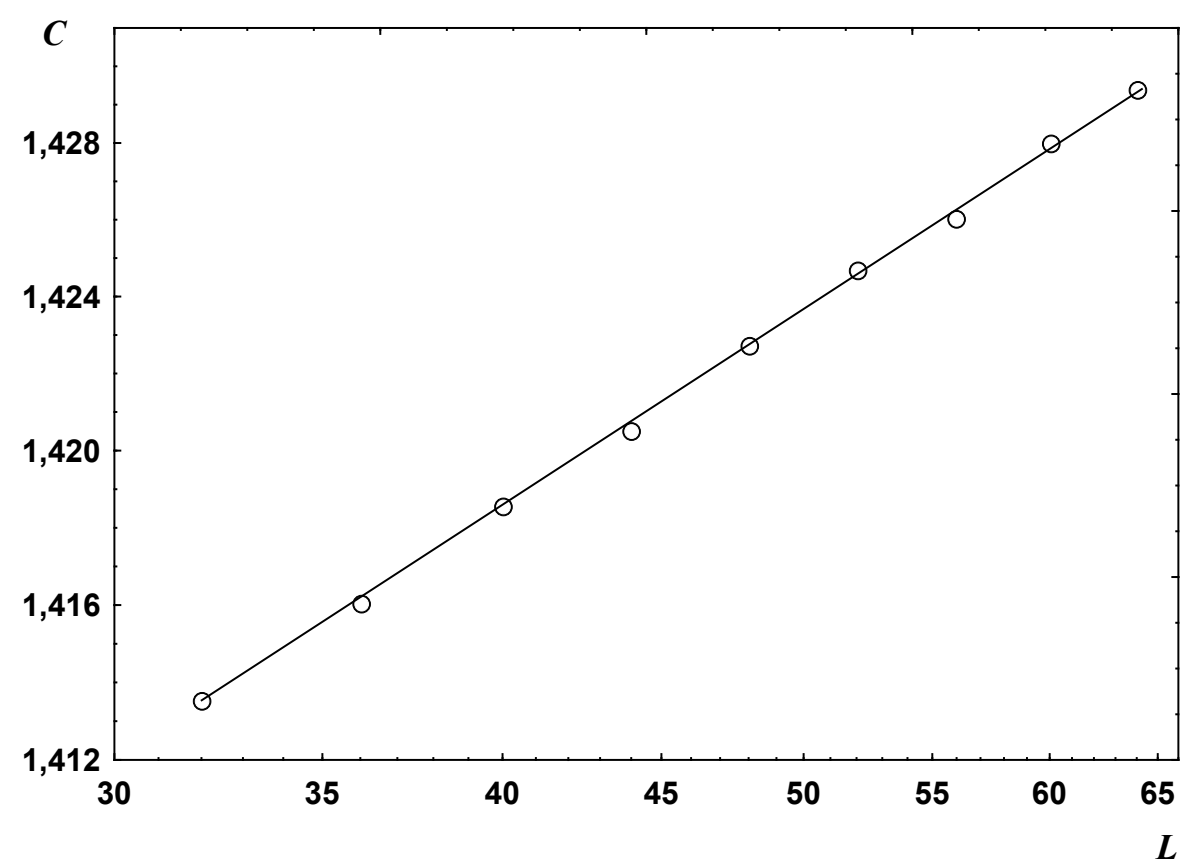

Figure 7. The log-log dependence of the heat capacity. 


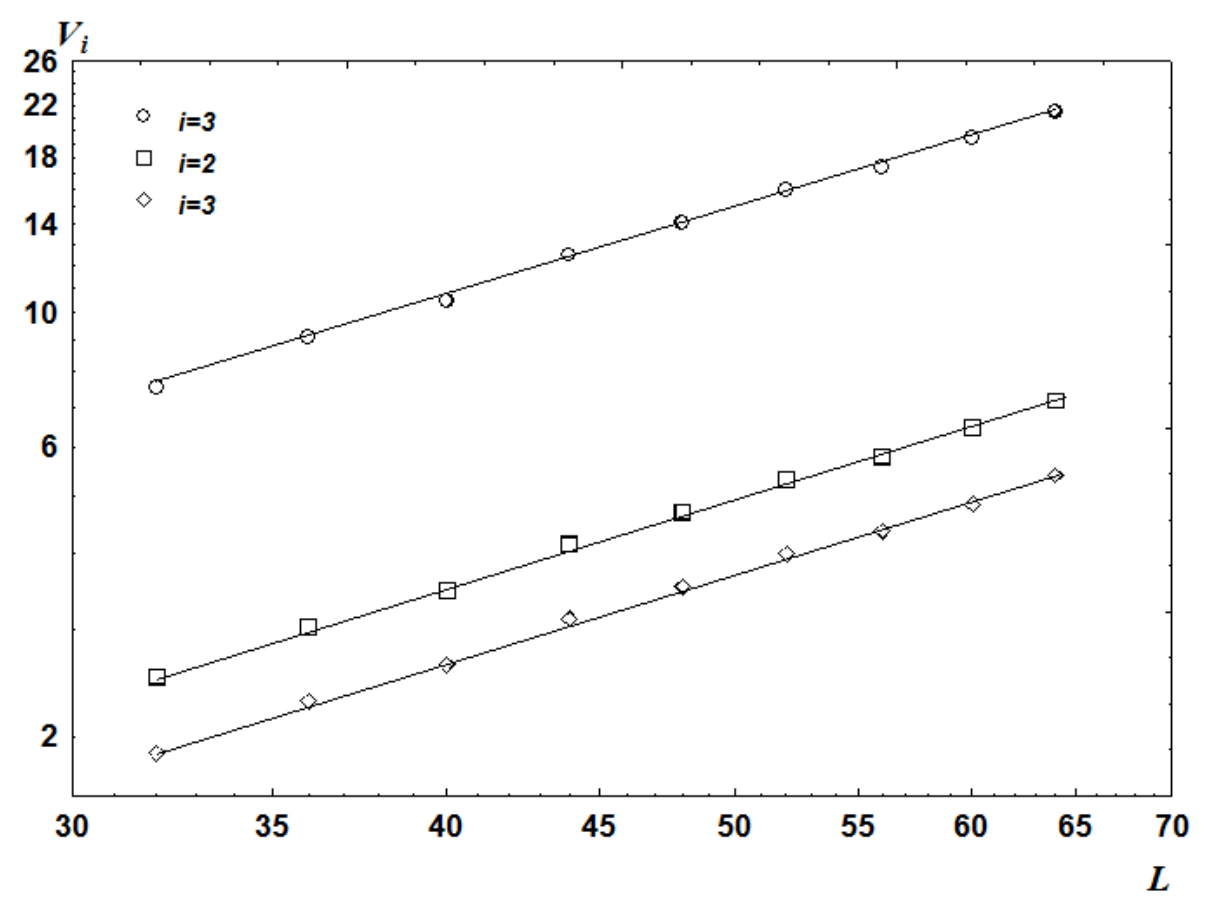

Figure 8. The log-log dependence of $V_{i}$ on linear sizes of the system.

Table 1. The values of the critical parameters of ANNNI - model.

\begin{tabular}{|c|c|c|c|c|c|c|c|c|c|}
\hline$\left|\mathbf{J}_{\mathbf{1}} / \mathbf{J}\right|$ & $\mathbf{T}_{\mathbf{c}}$ & $\boldsymbol{\alpha} / \boldsymbol{v}$ & $\boldsymbol{\beta} / \boldsymbol{v}$ & $\boldsymbol{\gamma} / \boldsymbol{v}$ & $\mathbf{1 / v}$ & $\boldsymbol{\alpha}$ & $\boldsymbol{\beta}$ & $\boldsymbol{\gamma}$ & $\boldsymbol{\nu}$ \\
\hline 0,271 & $3,736(1)$ & 0,352 & 0,431 & 2,499 & 1,799 & $0,196(4)$ & $0,240(4)$ & $1,389(6)$ & $0,556(6)$ \\
\hline 0,272 & 3,732 & 0,379 & 0,429 & 2,719 & 1,903 & 0,199 & 0,225 & 1,427 & 0,525 \\
\hline 0,273 & 3,728 & 0,420 & 0,422 & 2,880 & 2,055 & 0,203 & 0,206 & 1,403 & 0,487 \\
\hline 0,274 & 3,724 & 0,460 & 0,421 & 3,053 & 2,183 & 0,211 & 0,193 & 1,398 & 0,458 \\
\hline 0,275 & 3,726 & 0,449 & 0,422 & 3,002 & 2,125 & 0,211 & 0,199 & 1,414 & 0,471 \\
\hline 0,276 & 3,727 & 0,461 & 0,422 & 3,117 & 2,225 & 0,207 & 0,189 & 1,400 & 0,449 \\
\hline 0,30 & $3,723(3)$ & 0,142 & 0,447 & 2,109 & 1,609 & $0,088(4)$ & $0,278(4)$ & $1,312(6)$ & $0,622(6)$ \\
\hline 0,40 & 3,602 & 0,113 & 0,472 & 1,919 & 1,613 & 0,059 & 0,293 & 1,190 & 0,620 \\
\hline 0,50 & 3,672 & 0,101 & 0,492 & 1,980 & 1,550 & 0,065 & 0,317 & 1,277 & 0,645 \\
\hline 0,60 & 3,854 & 0,060 & 0,501 & 1,953 & 1,493 & 0,040 & 0,336 & 1,309 & 0,670 \\
\hline 0,70 & 3,976 & 0,011 & 0,516 & 1,967 & 1,492 & 0,007 & 0,346 & 1,318 & 0,670 \\
\hline 0,80 & 4,152 & 0,012 & 0,522 & 1,971 & 1,489 & 0,008 & 0,350 & 1,323 & 0,671 \\
\hline 0,90 & 4,334 & 0,011 & 0,509 & 1,965 & 1,490 & 0,007 & 0,342 & 1,319 & 0,671 \\
\hline 1,00 & 4,455 & 0,014 & 0,514 & 1,970 & 1,502 & 0,009 & 0,342 & 1,312 & 0,666 \\
\hline XY & & & & & & $-0,012$ & 0,3485 & 1,315 & 0,671 \\
\hline Ising & & & & & & 0,125 & 0,312 & 1,250 & 0,642 \\
\hline
\end{tabular}

According to data from the Table 1, it can be asserted that the closeness of a multicritical region influences on the critical exponent values for $\left|J_{l} / J\right| \leq 0,4$ [23], the critical exponent values of magnetization continue in growing and the critical exponents of susceptibility continue in decrease. With increase in the absolute value of $\left|J_{I} / J\right|$, the behavior of critical exponents of the magnetization and susceptibility brings nearer the system to the critical behavior, which is characteristic for the XY universality class of the critical behavior. A change in the critical exponent of the correlation radius also confirms a transition of the system from multicritical behavior into the typical behavior of $\mathrm{XY}$ universality class. But the critical exponent values of the heat capacity are impossible to describe by known universality classes. These critical exponents correspond by the sign to the critical behavior of Ising universality class, but by the absolute values are close to the XY universality class.

\section{Conclusions}

The obtained results demonstrate that the critical behavior 
of most systems differs from the predicted for corresponding universality classes. The reason for such a behavior is various anisotropic and competing interactions existing in the real systems. The account of all possible kinds of anisotropy and interactions creates the various types of the magnetic ordering in the system and, as a consequence, the multicritical phenomena, what results in complication of both thermodynamic and critical behavior of the system. Therefore, the study of such phenomena is also an important requirement in the investigation of critical phenomena. The development of new investigating methods and simulation algorithms for multicritical phenomena can promote the study not only of the neighborhood of Lifshitz point but extend the application of Monte-Carlo methods in research of more complex systems.

\section{REFERENCES}

[1] P.A. Fleury, Science 211 (1981) 125-131.

[2] M.A. Anisimov, E.E. Gorodetskii, V.M. Zaprudskii, Physics Uspekhi 24 (1981) 57-75.

[3] Yu.A. Izumov, V.M. Siromiatnikov, Phase transition and symmetry crystal, Moscow, Science 1984.

[4] W. Selke, Phys. Rep. 170 (1988) 213-264.

[5] R.J. Elliott, Phys. Rev. 124 (1961) 346.

[6] J. Yeomans, E. Henry, and T. David, in Solid State Physics.: Academic Press 41 (1988)151.

[7] W. Selke, Spatially modulated structures in systems with competing interactions In: Phase transitions and critical phenomena, Vol. 15, eds. C. Domb and J.L. Lebowitz (Academic Press), 1992, p.1-72

[8] W. Selke, M.E. Fisher, Phys. Rev. B 20 (1979) 257-265.

[9] R.M. Hornreich, M. Luban, S. Shtrikman, Phys. Rev. Lett. 35 (1975) 1678.

[10] A. K. Murtazaev, Zh. G. Ibaev, Low Temp. Phys. 35 (2009) 792-797.

[11] T. Garel and P. Pfeuty, J. Phys. C. 9 (1976) L245.

[12] S. Redner and H. E. Stanley, Phys. Rev. B. 16 (1977) 4901.
[13] M. E. Fisher and W. Selke, 302 (1981) 1-44

[14] P. Peszak, A. M. Ferrenberg, D. P. Landau, Phys. Rev. B 43 (1991) 6087.

[15] P. Bak, J. Boehm, Phys. Rev. B 21 (1980) 5297.

[16] A. Surda, Phys. Rev. B. 69 (2004) 134116.

[17] A. Gendiar and T. Nishino, Phys. Rev. B. 71024404 (2005).

[18] W. Selke and M. E. Fisher, J. Magn. Magn. Mat. 15-18 (1980) 403-404.

[19] E. B. Rasmussen, S. J. Knak-Jensen, Phys. Rev. B. 24 (1981) 2744-2750.

[20] K. Kaski and W. Selke, Phys. Rev. B 31 (1985) 3128-3131.

[21] M. Pleimling and M. Henkel, Phys. Rev. Lett. 87 (2001) 125702.

[22] A. K. Murtazaev, Zh. G. Ibaev, Journal of Experimental and Theoretical Physics 113 (2011) 106-112.

[23] A.K. Murtazaev, J.G. Ibaev, Solid State Communications 152 (2012) $177-179$.

[24] W. Selke, Z. Physik B 29 (1978) 133-137.

[25] J. Oitmaa, J. Phys. A: Math. Gen. 18 (1985) 365.

[26] Y. Muraoka, T. Kasama and T. Idogaki, J. Magn. and Magn. Mat. 272-276 (2004) E995-E996.

[27] A. K.Murtazaev, Zh.G. Ibaev, Journal of Experimental and Theoretical Physics 116 (2013) 266-271.

[28] U. Wolf, Phys. Rev. Lett. 62 (1989) 361.

[29] M. Henkel and M. Pleimling, Comp. Phys. Commun. 147 (2002) 161.

[30] R.J. Elliot, Phys. Rev. 124, (1961) 346.

[31] F. Wang and D.P. Landau, Phys. Rev. Lett. 47 (1981) 693.

[32] A. Mailhot, M.L. Plumer, and A. Caille, Phys. Rev. B 50 (1994-II) 6854.

[33] P. Peczak, A.M. Ferrenberg and D.P. Landau, Phys. Rev. B 43 (1991) 6087.

[34] D. Loison, Phys. Lett. A257 (1999) 83-87.

[35] A.K. Murtazaev, I.K. Kamilov, K.K. Aliev, J. Magn. and Magn. Mat. 204 (1999) 151-158. 